\title{
LR Number of Spherical Closed Curves
}

\author{
Kuniyuki TAKAOKA \\ Waseda University \\ (Communicated by K. Matsuzaki)
}

\begin{abstract}
For a given oriented spherical closed curve with $n$ transversal double points, we assign a cyclic word of length $2 n$ on two letters $L$ standing left and $R$ standing right by reading the crossing sign so that each crossing point is read once $L$ and once $R$. The LR number of the curve is the number of appearance of subwords $L R$ in the cyclic word. We completely determine oriented spherical closed curves whose LR numbers are less than or equal to three.
\end{abstract}

\section{Introduction}

In knot theory, knot diagrams play an important role to study and classify knots. In 2012, Higa, Nakanishi, Satoh and Yamamoto defined an OU sequence for a knot diagram, where the OU sequence is obtained from crossing information by reading a sequence of overand under- crossing points along the orientation direction of a knot [1]. They mainly studied about sequences which are realized by diagrams of the trefoil knot. After their study, K. Taniyama suggested the author to study LR sequences of knot diagrams. The LR sequence of a knot diagram is obtained from crossing information by reading a sequence of left- and right- crossing points along the orientation direction. The precise definition is given later. The LR sequence does not reflect any over/under crossing information. Therefore we define an LR sequence for an oriented closed curve on the 2-sphere with only finitely many transversal double points. We define an LR number of an LR sequence and completely determine oriented closed curves on the 2-sphere whose LR numbers are less than or equal to three. We note that there is a related study [2].

Let $L$ and $R$ be symbols. An $L R$ sequence of length $l \in \mathbf{Z}_{\geq 1}$ is a map $\varphi$ : $\{1,2, \ldots, l\} \longrightarrow\{L, R\}$. Such a sequence is encoded by $\varphi(1) \varphi(2) \cdots \varphi(l)$. We define a cyclic permutation $\rho:\{1,2, \ldots, l\} \longrightarrow\{1,2, \ldots, l\}$ by $\rho(i)=i+1(i=1,2, \ldots, l-1)$ and $\rho(l)=1$.

Let $\varphi_{1}$ and $\varphi_{2}$ be LR sequences with the same length. If there exists $i \in \mathbf{Z}$ such that $\varphi_{2}=$ $\varphi_{1} \circ \rho^{i}$, then we say that $\varphi_{1}$ and $\varphi_{2}$ are equivalent and denote it by $\varphi_{1} \sim \varphi_{2}$. It is clear that this is an equivalence relation. We do not distinguish an LR sequence and its equivalence class so

Received May 20, 2014; revised October 24, 2014

Key words: Spherical closed curve, LR word, LR number 


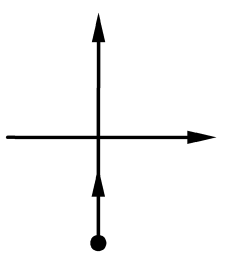

L

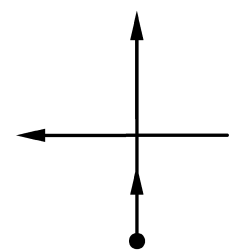

R

FIGURE 1

long as no confusion occurs. Let $S_{2 l}$ be the set of LR sequences of length $2 l$ with $\sharp \varphi^{-1}(L)=$ $\sharp \varphi^{-1}(R)=l$. The elements of the quotient set $S_{2 l} / \sim$ are called $L R$ words. For convenience, a consecutive sequence of $m\left(m \in \mathbf{Z}_{\geq 1}\right)$ copies of $L$ (resp. $\left.R\right)$ is denoted by $L^{m}$ (resp. $\left.R^{m}\right)$. Then an LR word $w$ of length $2 l$ can be written as $w=L^{\alpha_{1}} R^{\beta_{1}} L^{\alpha_{2}} R^{\beta_{2}} \cdots L^{\alpha_{n}} R^{\beta_{n}}$, where $\alpha_{1}, \alpha_{2}, \ldots, \alpha_{n}$ and $\beta_{1}, \beta_{2}, \ldots, \beta_{n}$ are positive integers with $\alpha_{1}+\alpha_{2}+\cdots+\alpha_{n}=$ $\beta_{1}+\beta_{2}+\cdots+\beta_{n}=l$. Note that

$$
\begin{aligned}
w & =L^{\alpha_{1}} R^{\beta_{1}} L^{\alpha_{2}} R^{\beta_{2}} \cdots L^{\alpha_{n}} R^{\beta_{n}} \\
& =L^{\alpha_{1}-1} R^{\beta_{1}} L^{\alpha_{2}} R^{\beta_{2}} \cdots L^{\alpha_{n}} R^{\beta_{n}} L \\
& =\cdots \\
& =R^{\beta_{1}} L^{\alpha_{2}} R^{\beta_{2}} \cdots L^{\alpha_{n}} R^{\beta_{n}} L^{\alpha_{1}} \\
& =R^{\beta_{1}-1} L^{\alpha_{2}} R^{\beta_{2}} \cdots L^{\alpha_{n}} R^{\beta_{n}} L^{\alpha_{1}} R \\
& =\cdots .
\end{aligned}
$$

Then the LR number of $w$, denoted by $\operatorname{lr}(w)$, is defined to be $n$.

We consider an oriented closed curve with finitely many transversal double points without any other singularities in the two-dimensional sphere $S^{2}$. In this paper, by an oriented spherical closed curve, we mean such a curve. We consider it up to orientation preserving auto-homeomorphisms on $S^{2}$. A transversal double point is called a crossing point.

We take a base point except for the crossing points on an oriented spherical closed curve $P$ and trace $P$ along the orientation direction. When we pass a crossing point, the crossing is read to be left (resp. right) if the curve that one crosses travel from left to right (resp. from right to left). Then we record the symbol $L$ (resp. $R$ ) if the crossing is left (resp. right) at each crossing point (see Fig. 1). We continue this recording until we return to the base point.

Note that we pass each crossing point twice, and once it is read $L$ and once $R$. Therefore we obtain an LR word from the crossing information on $P$ by reading a sequence of leftand right- crossing along the orientation direction of $P$. It is called an $L R$ word for $P$ and denoted by $w_{P}$. We define the $L R$ number of $P$, denoted by $\operatorname{lr}(P)$, to be the LR number of $w_{P}$. Namely $\operatorname{lr}(P)=\operatorname{lr}\left(w_{p}\right)$. See for example Fig. 2 . 


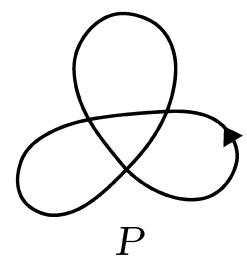

FIGURE 2. $w_{P}=L R L R L R, \operatorname{lr}(P)=3$.

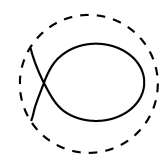

FIGURE 3

We define the LR word for a simple closed curve in $S^{2}$ to be the empty word $\varepsilon$ and $\operatorname{lr}(\varepsilon)$ to be zero.

For any $P$, we obtain an LR word for $P$. Conversely, given an LR word $w$, we can construct a spherical closed curve whose LR word coincides with $w$ (Proposition 2.2). Therefore, for any positive integer $n$, there exists an oriented spherical closed curve whose LR number is $n$.

A spherical closed curve $P$ is said to be prime if for any simple closed curve $C$ in $S^{2}$ which intersects $P$ transversally in two points, exactly one of subcurves of $P$ cut by $C$ is a simple arc. A spherical closed curve $P$ is said to be reduced if $P$ does not have subcurves illustrated in Fig. 3.

The main results of this paper are Theorems 2.4, 3.2, 3.5 and 3.8. Theorem 2.4 is proved in Section 2. In Section 3, by Theorem 2.4, we describe all prime oriented spherical closed curves whose LR numbers are less than or equal to three (Theorems 3.2, 3.5 and 3.8). Then we describe all non-prime oriented spherical closed curves whose LR numbers are less than or equal to three (Theorem 3.2, Corollaries 3.10 and 3.11).

\section{A concentric circular curve}

Let $w$ be an LR word. Assume that $w=u_{1} L R u_{2}$ or $w=u_{1} R L u_{2}$, where $u_{1}$ and $u_{2}$ are LR subsequences. Then we say that an LR word $w^{\prime}=u_{1} u_{2}$ is obtained from $w$ by a contraction (cf. [1]).

LEMMA 2.1. Let $w$ be an LR word, and $w^{\prime}$ an LR word obtained from $w$ by a contraction. If there exists an oriented spherical closed curve $P^{\prime}$ with $w_{P^{\prime}}=w^{\prime}$, then there exists an oriented spherical closed curve $P$ with $w_{P}=w$. 


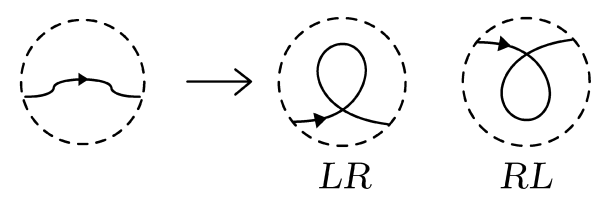

FIGURE 4
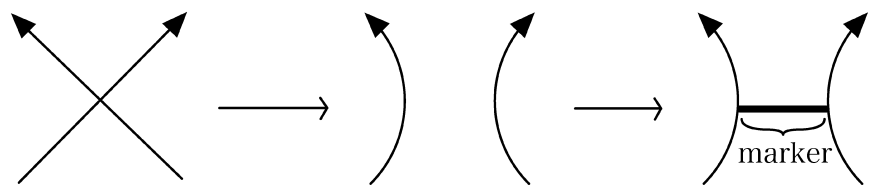

FIGURE 5. A smoothing, then giving a marker to a crossing point.

Proof. Following the similar lines as [1], we make a local change illustrated in Fig. 4 to the arc of $P^{\prime}$ corresponding to the LR subsequence $L R$ or $R L$ contracted. Then we obtain a new oriented spherical closed curve $P$ with $w_{P}=w$.

PROPOSITION 2.2. For any LR word $w$, there exists an oriented spherical closed curve $P$ such that $w_{P}=w$.

PROOF. For any LR word $w$, there exists an LR subsequence $L R$ or $R L$ in $w$. By the induction on the length of $w$, we obtain an empty word $\varepsilon$ from $w$ by a finite sequence of contractions. Since $\varepsilon$ corresponds to a simple closed curve in $S^{2}$, we obtain an oriented spherical closed curve whose LR word coincides with $w$ inductively by Lemma 2.1 .

Let $P$ be an oriented spherical closed curve. In a small neighborhood of each crossing point of $P$, we make the following local change to $P$ : delete the crossing point and connect the ends in the only way compatible with orientation as in Fig. 5. We call this local change a smoothing.

When the smoothing has been done at all crossing points, $P$ becomes a set of disjoint simple closed curves in $S^{2}$. We call these closed curves Seifert circles. Applying smoothings to all crossing points of $P$ and giving line segments called markers on the sites of crossing points, we obtain a figure called a Seifert diagram for $P$, which is denoted by $S_{d}(P)$. The Seifert diagram for $P$ is constructed by the Seifert circles of $P$ and the markers corresponding to the crossing points of $P$.

In the same way as Section 1, we will obtain the LR word for $S_{d}(P)$. First we determine how to circulate $S_{d}(P)$ in the following: tracing a Seifert circle $\gamma$ of $S_{d}(P)$, if we encounter a marker on the left- (resp. right-) hand side for the direction of travelling, then we leave $\gamma$, move along the marker and go into a Seifert circle on the left- (resp. right-) hand side of $\gamma$ 


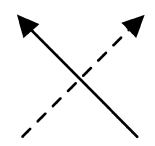

$\mathrm{L}$

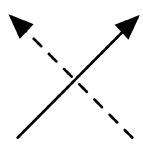

$\mathrm{R}$

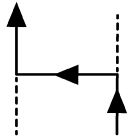

$\mathrm{L}$

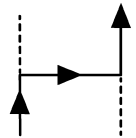

$\mathrm{R}$

FIGURE 6. A left (resp. right) crossing point corresponds to the left (resp. right) marker.

along the marker. A marker is said to be left (resp. right) for $\gamma$ if we pass the marker and take to a Seifert circle on left- (resp. right-) hand side of $\gamma$. A left marker is labeled $L$ and a right marker $R$ (see Fig. 6).

Then, circulating $S_{d}(P)$, we obtain the LR word from marker information of $S_{d}(P)$. Notice that since we can restore $S_{d}(P)$ to $P$, the LR word for $S_{d}(P)$ coincides with $w_{P}$. Therefore we may treat $S_{d}(P)$ instead of $P$ in some cases.

Let $\mathcal{S}(P)$ be the set of Seifert circles of $P$ in $S^{2}$. Define

$$
\begin{aligned}
I_{+}(\mathcal{S}(P)) & :=\{\gamma \in \mathcal{S}(P) \mid \gamma \text { is a clockwise innermost circle in } \mathcal{S}(P)\}, \\
I_{-}(\mathcal{S}(P)) & :=\{\gamma \in \mathcal{S}(P) \mid \gamma \text { is a counterclockwise innermost circle in } \mathcal{S}(P)\}, \\
I(\mathcal{S}(P)) & :=\{\gamma \in \mathcal{S}(P) \mid \gamma \text { is an innermost circle in } \mathcal{S}(P)\} .
\end{aligned}
$$

Then we have $I(\mathcal{S}(P))=I_{+}(\mathcal{S}(P)) \sqcup I_{-}(\mathcal{S}(P))$.

We say that $P$ is a concentric circular curve if $\sharp I_{+}(\mathcal{S}(P))=\sharp I_{-}(\mathcal{S}(P))=1$.

Let $c(\gamma)$ be the number of markers which the innermost circle $\gamma$ touches. We define

$$
i_{c_{+}}(P):=\sum_{\gamma \in I_{+}(\mathcal{S}(P))} c(\gamma), \quad i_{c_{-}}(P):=\sum_{\gamma \in I_{-}(\mathcal{S}(P))} c(\gamma) .
$$

LEMMA 2.3. Let $P$ be an oriented spherical closed curve. Then

(1) $\operatorname{lr}(P) \geq i_{c_{+}}(P)$,

(2) $\operatorname{lr}(P) \geq i_{c_{-}}(P)$ and

(3) $\operatorname{lr}(P) \geq \max \left\{i_{c_{+}}(P), i_{c_{-}}(P)\right\}$.

Proof. (1) Let $S_{d}(P)$ be the Seifert diagram for $P$, and $m_{1}, m_{2}, \ldots, m_{n}$ the markers which a clockwise innermost circle $\gamma$ touches in $S_{d}(P)$ (see Fig. 7). Pass through $m_{i}$, trace the arc of $\gamma$ and pass again through $m_{i+1}\left(m_{1}\right.$ when $\left.i=n\right)$, then we have an LR subsequence $R L$ obtained from the marker information.

Hence, if a clockwise innermost circle touches $n$ markers, then the LR word for $S_{d}(P)$ contains at least $n$ LR subsequences $R L$. Note that no two clockwise innermost circles touch a common marker. Therefore $\operatorname{lr}(P) \geq i_{c_{+}}(P)$.

By a similar argument, we have (2). Then (3) follows immediately.

THEOREM 2.4. Let $P$ be a reduced oriented closed spherical curve. If the LR number of $w_{P}$ is less than or equal to three, then $P$ is a concentric circular curve. 


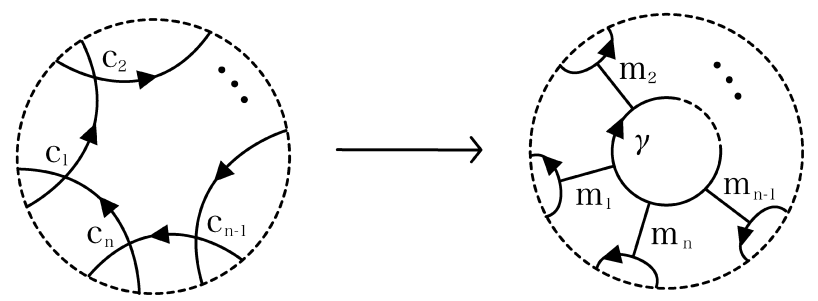

FIGURE 7. To each crossing point $c_{i}(1 \leq i \leq n)$, we apply smoothing and giving a maker.

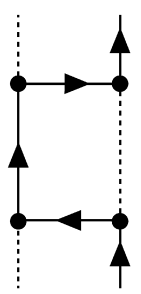

type A

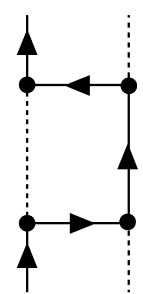

type B

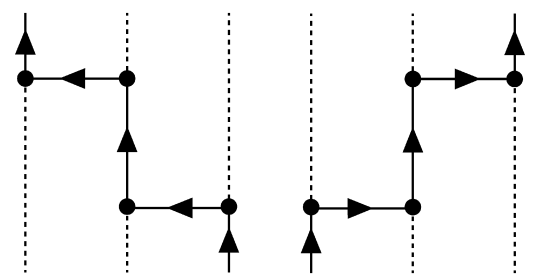

type $\mathrm{C}$

FIGURE 8

PROOF. If $P$ is not a concentric circular curve, then $\sharp I(\mathcal{S}(P)) \geq 3$. Namely, $\sharp I_{+}(\mathcal{S}(P)) \geq 2$ or $\sharp I_{-}(\mathcal{S}(P)) \geq 2$. On the other hand, since $P$ is reduced, we have $c(\gamma) \geq 2$ for all $\gamma \in I(\mathcal{S}(P))$. Therefore $i_{c_{+}}(P) \geq 4$ or $i_{c_{-}}(P) \geq 4$. Thus $\operatorname{lr}(P) \geq 4$ by Lemma 2.3 .

\section{Spherical closed curves with LR number 1,2 or 3}

In this section we determine prime oriented spherical closed curves whose LR numbers are less than or equal to three. We need some lemmas in order to construct such curves.

Let $P$ be a concentric circular curve in $S^{2}$, and $S_{d}(P)$ the Seifert diagram for $P$. There are four patterns of the parts of the circulation of $S_{d}(P)$ as illustrated in Fig. 8. They are between two or three adjacent Seifert circles of $S_{d}(P)$.

A pattern on the left-hand side of Fig. 8 is called type A, one on the second from the left type $B$, and the others type $C$. We obtain an LR subsequence $L R$ or $R L$ from marker information of type $\mathrm{A}$ or $\mathrm{B}$, and $L L$ or $R R$ from that of type C. Then the LR number of $S_{d}(P)$ is $n$ if and only if $S_{d}(P)$ has exactly $n$ type A patterns, and then $S_{d}(P)$ has exactly $n$ type B patterns. Therefore we may count the number of patterns of type A or B in $S_{d}(P)$ to calculate the LR number of $P$.

Suppose that $S_{d}(P)$ has $k$ Seifert circles $\gamma_{1}, \ldots, \gamma_{k}$ where $k \geq 2$. Let $\gamma_{1}$ be the counter- 


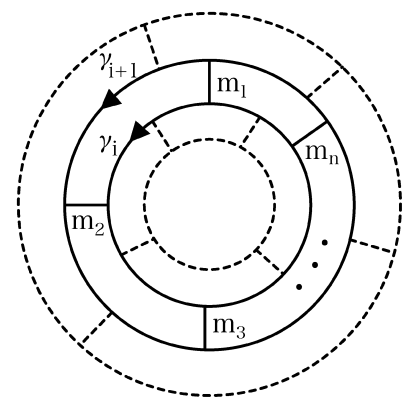

FIGURE 9. In the neighborhood of $\gamma_{i}$ and $\gamma_{i+1}$.

clockwise innermost circle, and $\gamma_{k}$ the clockwise innermost circle. Assume that $\gamma_{i}$ and $\gamma_{i+1}$ are adjacent $(1 \leq i \leq k-1)$. Such Seifert circles are called the Seifert circles of $S_{d}(P)$ arranged in order.

LEMMA 3.1. Let $P$ be a concentric circular curve, and $S_{d}(P)$ the Seifert diagram for $P$. Let $\gamma_{1}, \ldots, \gamma_{k}$ be the Seifert circles of $S_{d}(P)$ arranged in order where $k \geq 2$. If there exists $\gamma_{i}(1 \leq i \leq k-1)$ such that the number of the markers between $\gamma_{i}$ and $\gamma_{i+1}$ is $n$, then the LR number of $P$ is greater than or equal to $n$.

Proof. Let $m_{1}, \ldots, m_{n}$ be $n$ markers between $\gamma_{i}$ and $\gamma_{i+1}$ (see Fig. 9).

Assume that we leave $\gamma_{i}$, pass $m_{1}$ and take to $\gamma_{i+1}$ when circulating the $S_{d}(P)$. Since $m_{1}$ is a right marker for $\gamma_{i}$, we obtain an LR subsequence $R$. In order to turn back to $\gamma_{i}$ again, we need to pass $m_{h}(1 \leq h \leq n)$ in direction of $\gamma_{i}$ once. At this time, $m_{h}$ is a left marker for $\gamma_{i+1}$. Then we obtain an LR subsequence $\varphi$ of length $l(l \geq 2)$, which contains at least one $R$ and one $L$ in this process. Therefore there exists $j(j<l)$ such that $\varphi(j)=R$ and $\varphi(j+1)=L$. This shows that we have a type B pattern in the area between $\gamma_{i}$ and $\gamma_{k}$.

Applying a similar argument for each marker, we have at least $n$ type B patterns in the area between $\gamma_{i}$ and $\gamma_{k}$. Therefore $\operatorname{lr}(P) \geq n$.

THEOREM 3.2. Let $P$ be an oriented spherical closed curve. If the LR number of $P$ is one, then $P$ is one of the following curves illustrated in Fig. 10. In particular a spherical closed curve in the far left-hand side of Fig. 10 is prime and the others are not prime.

Proof. By Lemma 2.3, $i_{c_{+}}(P)=i_{c_{-}}(P)=1$. Thus $\sharp I_{+}(\mathcal{S}(P))=\sharp I_{-}(\mathcal{S}(P))=1$, that is, $P$ is a concentric circular curve. Let $\gamma_{1}, \ldots, \gamma_{k}$ be the Seifert circles of $S_{d}(P)$ arranged in order where $k \geq 2$. By Lemma 3.1, we see that for any $\gamma_{i}(1 \leq i \leq k-1)$, the number of markers between $\gamma_{i}$ and $\gamma_{i+1}$ is one. Therefore we obtain the following Seifert diagrams for $P$ illustrated in Fig. 11. 

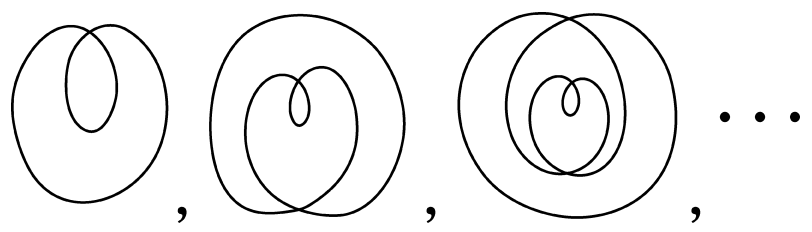

FIGURE 10
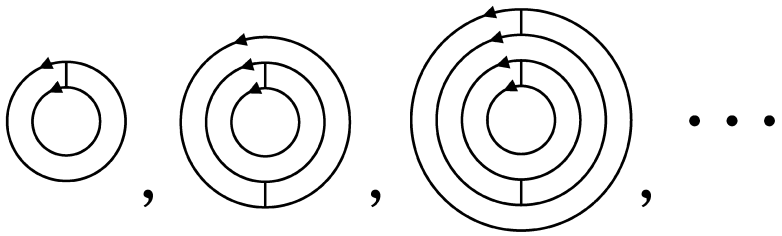

FIGURE 11

Lemma 3.3. Let $P$ be a concentric circular curve, and $S_{d}(P)$ a Seifert diagram for $P$. Let $\gamma_{1}, \ldots, \gamma_{k}$ be the Seifert circles of $S_{d}(P)$ arranged in order where $k \geq 3$. If there exists $\gamma_{i}(1 \leq i \leq k-1)$ such that the number of the markers between $\gamma_{i}$ and $\gamma_{i+1}$ is one, then $P$ is not prime.

PROOF. The marker between $\gamma_{i}$ and $\gamma_{i+1}$ represents a crossing point $c$ in $P$ such that $P \backslash\{c\}$ is disconnected. Therefore $P$ is not prime.

Let $\gamma_{1}, \ldots, \gamma_{k}$ be the Seifert circles of $S_{d}(P)$ arranged in order where $k \geq 3$. Let $m_{1}^{i}, \ldots, m_{p}^{i}$ be $p$ markers between $\gamma_{i}$ and $\gamma_{i+1}$, and $m_{1}^{i-1}, \ldots, m_{q}^{i-1} q$ markers between $\gamma_{i-1}$ and $\gamma_{i}$ where $2 \leq i \leq k-1$ and $p$ and $q$ are positive integers. We define the endpoints of the marker

$$
e_{h}^{i}:=m_{h}^{i} \cap \gamma_{i}(1 \leq h \leq p), \quad e_{j}^{i-1}:=m_{j}^{i-1} \cap \gamma_{i}(1 \leq j \leq q) .
$$

Tracing $\gamma_{i}$, we obtain a cyclic sequence of endpoints. We denote it by $E\left(\gamma_{i}\right)$.

LEMMA 3.4. Let $P$ be a concentric circular curve, and $S_{d}(P)$ the Seifert diagram for $P$. Let $\gamma_{1}, \ldots, \gamma_{k}$ be the Seifert circles of $S_{d}(P)$ arranged in order where $k \geq 3$. If there exists a Seifert circle $\gamma_{i}(2 \leq i \leq k-1)$ such that $E\left(\gamma_{i}\right)$ coincides $e_{1}^{i} e_{2}^{i} \cdots e_{p}^{i} e_{1}^{i-1} e_{2}^{i-1} \cdots e_{q}^{i-1}$ $(p, q \in \mathbf{N})$, then $P$ is not prime.

Proof. There exists a simple closed curve $\alpha$ that intersects $S_{d}(P)$ transversally in two points as illustrated in Fig. 12. Therefore $P$ restored from $S_{d}(P)$ is not prime.

Now we construct prime oriented spherical closed curves whose LR numbers are two. 


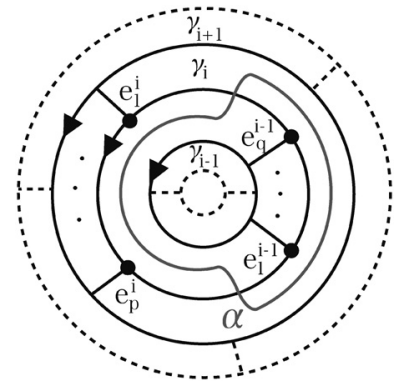

FIGURE 12

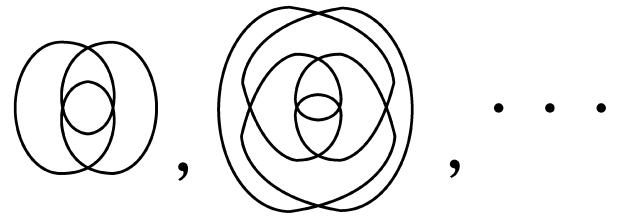

FIGURE 13
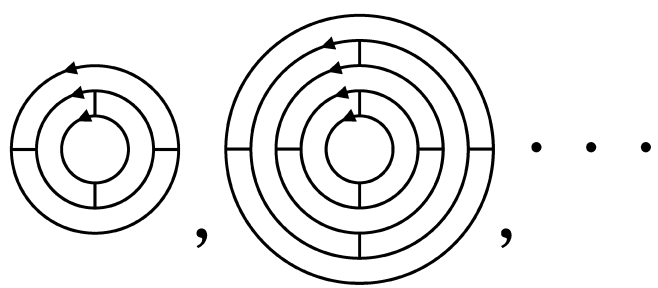

FIGURE 14

THEOREM 3.5. Let $P$ be a prime oriented spherical closed curve. If the LR number of $P$ is two, then $P$ is one of the following curves illustrated in Fig. 13.

PROOF. It is clear that prime spherical closed curves with two or more crossing points are reduced. Thus $P$ is a concentric circular curve by Theorem 2.4.

We deal with $S_{d}(P)$ instead of $P$. By Lemmas 3.1 and 3.3, any Seifert circles of $S_{d}(P)$ have just two markers. Furthermore, $S_{d}(P)$ does not contain the portions illustrated in Fig. 12 by Lemma 3.4. Therefore we obtain the following Seifert diagram for $P$ illustrated in Fig. 14.

Lastly we construct prime oriented spherical closed curves whose LR numbers are three. 


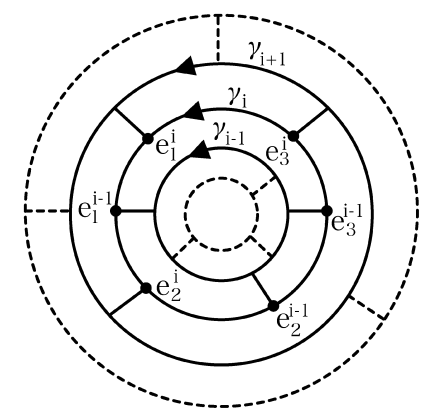

FIGURE 15. The case of $p=q=3$ and $E\left(\gamma_{i}\right)$ contains a sequence $e_{2}^{i-1} e_{3}^{i-1}$.

LEMMA 3.6. Let $P$ be a concentric circular curve, $S_{d}(P)$ the Seifert diagram for $P$, and $\gamma_{1}, \ldots, \gamma_{k}$ the Seifert circles of $S_{d}(P)$ arranged in order where $k \geq 3$. If there exists $\gamma_{i}$ $(2 \leq i \leq k-1)$ such that $S_{d}(P)$ has $p$ markers between $\gamma_{i}$ and $\gamma_{i+1}$ and $q$ markers between $\gamma_{i-1}$ and $\gamma_{i}$, and $E\left(\gamma_{i}\right)$ contains $j$ subsequences $e_{h}^{i-1} e_{h+1}^{i-1}(h, j \leq q-1)$, then the LR number of $P$ is greater than or equal to $p+j$.

PROOF. We will count the number of type B patterns in $S_{d}(P)$ to calculate the LR number of $P$.

Since the number of the markers between $\gamma_{i}$ and $\gamma_{i+1}$ is $p, S_{d}(P)$ has at least $p$ type $B$ patterns in the area between $\gamma_{i}$ and $\gamma_{k}$. Then $\operatorname{lr}(P) \geq p$. In addition, since $E\left(\gamma_{i}\right)$ contains $j$ subsequences $e_{h}^{i-1} e_{h+1}^{i-1}, S_{d}(P)$ has $j$ type $B$ patterns in the area between $\gamma_{i-1}$ and $\gamma_{i}$. Therefore $\operatorname{lr}(P) \geq p+j$.

LEMMA 3.7. Let $P$ be a concentric circular curve, $S_{d}(P)$ the Seifert diagram for $P$, and $\gamma_{1}, \ldots, \gamma_{k}$ the Seifert circles of $S_{d}(P)$ arranged in order where $k \geq 3$. If there exists $\gamma_{i_{1}}$ and $\gamma_{i_{2}}\left(2 \leq i_{2}<i_{1} \leq k-1\right)$ such that $S_{d}(P)$ has $p_{1}$ markers between $\gamma_{i_{1}}$ and $\gamma_{i_{1}+1}, p_{2}$ markers between $\gamma_{i_{2}}$ and $\gamma_{i_{2}+1}$, and $p_{3}$ markers between $\gamma_{i_{2}-1}$ and $\gamma_{i_{2}}\left(p_{1}>p_{2}, p_{3}>p_{2}\right)$, then the LR number of $P$ is greater than or equal to $p_{1}-p_{2}+p_{3}$.

PROOF. We will count the number of type B patterns in $S_{d}(P)$ to calculate the LR number of $P$. Since the number of the markers between $\gamma_{i_{1}}$ and $\gamma_{i_{1}+1}$ is $p_{1}, S_{d}(P)$ has at least $p_{1}$ type $B$ patterns in the area between $\gamma_{i_{1}}$ and $\gamma_{k}$. Then $\operatorname{lr}(P) \geq p_{1}$. In addition, $S_{d}(P)$ has $p_{3}-p_{2}$ type $B$ patterns in the area between $\gamma_{i_{2}-1}$ and $\gamma_{i_{2}}$. Therefore $\operatorname{lr}(P) \geq p_{1}-p_{2}+p_{3}$.

THEOREM 3.8. Let $P$ be a prime oriented spherical closed curve. If the LR number of $P$ is three, then $P$ is obtained from $T(3, n)$ by replacing one of the areas $A, B$ and $C$ with $S(2, l)$, and by replacing the area $D$ with $S(2, m)$ as in Fig. 16 where $n \geq 2$ and $l, m \geq 0$.

Proof. Since $P$ has more than two crossing points, $P$ is reduced. Thus, by Theorem 2.4, $P$ is a concentric circular curve. 


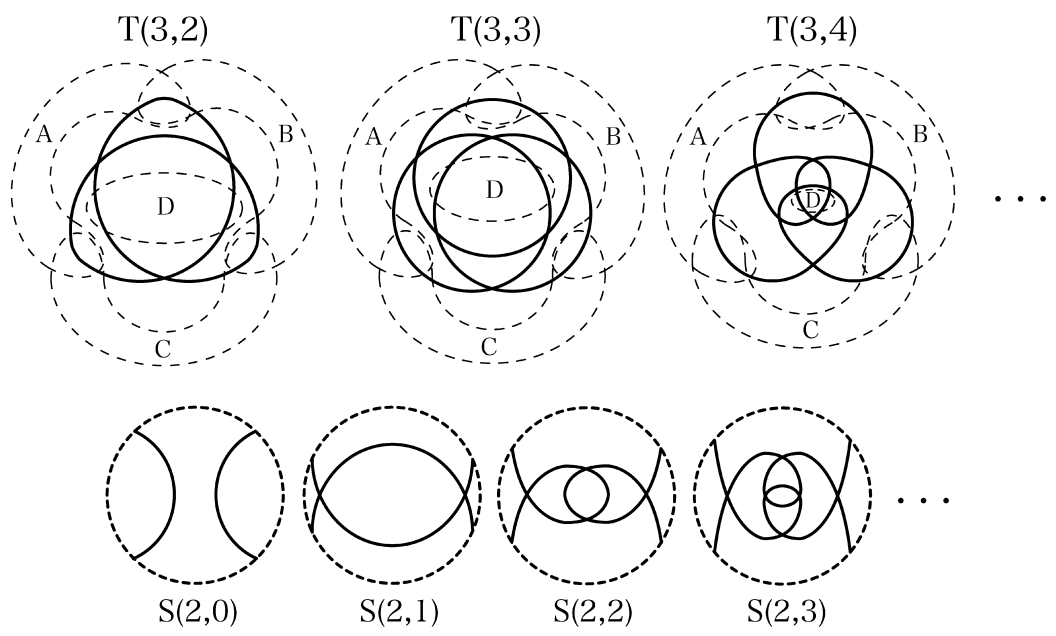

FIGURE 16

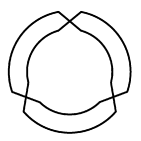

(1)

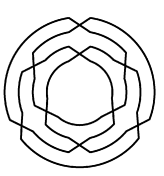

(2)

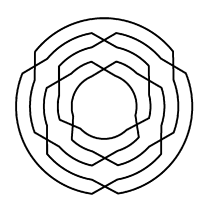

(3)

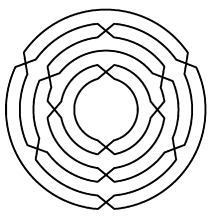

(4)

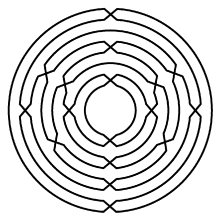

(5)

FIGURE 17

We deal with $S_{d}(P)$ instead of $P$. By Lemmas 3.1 and 3.3, any Seifert circles of $S_{d}(P)$ have two or three markers. In addition, by Lemma 3.4, $S_{d}(P)$ does not contain the portions illustrated in Fig. 12. Furthermore, $S_{d}(P)$ does not satisfy the conditions of Lemmas 3.6 and 3.7. By these facts, we have the desired conclusion.

Some examples of prime oriented spherical closed curves with LR number three are illustrated in Fig. 17. The curve (1) is $T(3,2),(2)$ is $T(3,4),(3)$ is $T(3,5),(4)$ is obtained from $T(3,4)$ by replacing $D$ with $S(2,2)$, and $(5)$ is obtained from $T(3,4)$ by replacing both $C$ and $D$ with $S(2,2)$.

Let $P_{1}$ and $P_{2}$ be spherical closed curves. Suppose that $P_{1}$ and $P_{2}$ are disjoint. We make the following local change to $P_{1}$ and $P_{2}$. Find a disk $D \subset S^{2}$ such that $D \cap P_{i}=\partial D \cap P_{i}$ is a simple arc for $i=1,2$.

Let $P=\left(P_{1} \cup P_{2} \cup \partial D\right) \backslash \operatorname{int}\left(\partial D \cap\left(P_{1} \cup P_{2}\right)\right)$. Then $P$ is said to be a composition of $P_{1}$ and $P_{2}$. 


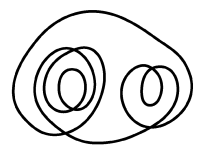

(1)

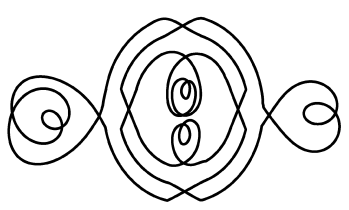

(2)

FIGURE 18. Examples of cases (1) and (2) of Corollary 3.10.

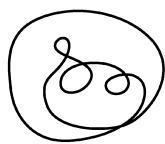

(1)

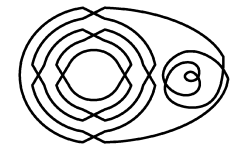

(2)

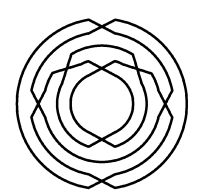

(3)

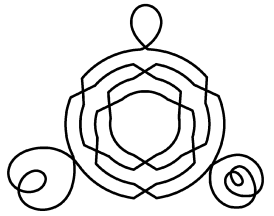

(4)

FIgURE 19. Examples of cases (1), (2), (3) and (4) of Corollary 3.11.

LEMMA 3.9. Let $P_{1}$ and $P_{2}$ be oriented spherical closed curves. If the LR number of $P_{1}$ is $n_{1}$ and that of $P_{2}$ is $n_{2}$, then the LR number of a composition of $P_{1}$ and $P_{2}$ is $n_{1}+n_{2}+1$, $n_{1}+n_{2}$ or $n_{1}+n_{2}-1$.

PROOF. Let $w_{P_{1}}$ be the LR word for $P_{1}$, and $w_{P_{2}}$ the LR word for $P_{2}$. The proof immediately follows by considering to put $w_{P_{2}}$ between the letters of $w_{P_{1}}$.

By Theorems 3.5 and 3.8, and Lemma 3.9, we describe how to construct non-prime oriented spherical closed curves whose LR numbers are two or three.

COROLlARY 3.10. Let $P$ be a non-prime oriented spherical closed curve whose LR number is two. Then $P$ is one of the following curves.

(1) The curve $P$ is a composition of two oriented spherical closed curves whose LR numbers are one.

(2) The curve $P$ is a composition of a prime oriented spherical closed curve whose LR number is two and at most four oriented spherical closed curves whose LR numbers are one.

COROLLARY 3.11. Let P be a non-prime oriented spherical closed curve whose LR number is three. Then $P$ is one of the following curves.

(1) The curve P is a composition of at most three oriented spherical closed curves whose LR numbers are one.

(2) The curve $P$ is a composition of an oriented spherical closed curve whose LR number is two and an oriented spherical closed curve whose LR number is one. 
(3) The curve $P$ is a composition of two oriented spherical closed curves whose LR numbers are two.

(4) The curve $P$ is a composition of a prime oriented spherical closed curve whose LR number is three and at most six oriented spherical closed curves whose LR numbers are one.

ACKnOwledgments. The author would like to thank Professor Kouki Taniyama for his fruitful suggestions and comments. The author is also grateful to Professor Noboru Ito and the referee for their helpful comments.

\section{References}

[ 1 ] R. Higa, Y. NakAnishi, S. SAtoh and T. YAmamoto, Crossing information and warping polynomials about the trefoil knot, Journal of Knot Theory and Its Ramifications 21, No. 12 (2012).

[2] R. Shinjo and K. TANAKA, On the inclusive relation of three properties of knot diagrams, Titles and Short Summaries of the Talks, MSJ 2012 autumn meeting at Kyushu University.

Present Address:

Department of Mathematics, Graduate School of Education, WASEDA UNIVERSITY,

1-6-1, Nishi-WASEDA, SHINJUKU-KU, TOKYO 169-8050, JAPAN.

e-mail: kuni.takaoka@akane.waseda.jp 